\title{
The averaging trick and the Černý conjecture
}

\author{
Benjamin Steinberg* \\ School of Mathematics and Statistics \\ Carleton University \\ 1125 Colonel By Drive \\ Ottawa, ON, Canada \\ bsteinbg@math.carleton.ca
}

\begin{abstract}
The results of several papers concerning the Černý conjecture are deduced as consequences of a simple idea that I call the averaging trick. This idea is implicitly used in the literature, but no attempt was made to formalize the proof scheme axiomatically. Instead, authors axiomatized classes of automata to which it applies.
\end{abstract}

\section{Introduction}

Recall that a (complete deterministic) automaton $\mathscr{A}=(Q, \Sigma)$ with state set $Q$ and alphabet $\Sigma$ is called synchronizing if there is a word $w \in \Sigma^{*}$ such that $|Q w|=1$. The word $w$ is called a synchronizing word. The main conjecture in this area is:

Conjecture 1 (Černý [1]) An n-state synchronizing automaton admits a synchronizing word of length at most $(n-1)^{2}$.

There is a vast literature on this subject. See for example [1-25]. The best known upper bound is cubic [26], whereas it is known that one cannot do better than $(n-1)^{2}[1]$.

My goal here in this note is not to prove the Černý conjecture for a new class of automata, but rather to give a no-frills, uniform approach to an argument that underlies a growing number of results in the Černý conjecture literature (cf. [7,13,20-22,24]). Underlying all these results (as well as the more difficult results of [5] and [17]) are two simple ideas:

- if a finite sequence of numbers is not constant, then it must at some place exceed its average;

- finite dimensional vector spaces satisfy the ascending chain condition on subspaces.

\footnotetext{
* The author gratefully acknowledges the support of NSERC
} 
The latter idea is often cloaked in the language of rational power series.

The paper is organized as follows. In the next section I state what I call the "Averaging Lemma." It is a method, with a probabilistic flavor, for obtaining bounds on lengths of synchronizing words. Before proving the lemma, I show how to deduce from it Kari's solution of the Cerný conjecture for Eulerian automata, as well as recent results of Béal and Perrin [20] for one-cluster automata and Carpi and d'Alessandro [21, 22] for (locally) strongly transitive automata. We also recover an old result of Rystsov [7] on regular automata (which is essentially the same thing as strongly transitive automata). In fact, we obtain new generalizations of all these results. The final section proves the Averaging Lemma.

\section{The averaging trick}

Let $\Sigma$ be an alphabet. Denote by $\Sigma^{*}$ the free monoid on $\Sigma$ and put

$$
\Sigma^{\leq d}=\bigcup_{m=0}^{d} \Sigma^{m} .
$$

The ring of polynomials with real coefficients in the non-commuting variables $\Sigma$ is denoted $\mathbb{R} \Sigma$. By a (finitely supported) probability on $\Sigma^{*}$, we mean an element

$$
P=\sum_{w \in \Sigma^{*}} P(w) w \in \mathbb{R} \Sigma
$$

such that: $P(w) \geq 0$ for all $w \in \Sigma^{*}$; and

$$
\sum_{w \in \Sigma^{*}} P(w)=1
$$

The support of $P$ is

$$
\sigma(P)=\left\{w \in \Sigma^{*} \mid P(w)>0\right\} .
$$

Notice that if $P_{1}$ and $P_{2}$ are probabilities, then so is $P_{1} P_{2}$. Also note that $\sigma\left(P_{1} P_{2}\right)=\sigma\left(P_{1}\right) \sigma\left(P_{2}\right)$.

If $X: \Sigma^{*} \rightarrow \mathbb{R}$ is a random variable, then the expected value of $X$ (with respect to the probability $P$ ) is:

$$
\mathbf{E}_{P}(X)=\sum_{w \in \Sigma^{*}} P(w) X(w)=\sum_{w \in \sigma(P)} P(w) X(w) .
$$


The fundamental property of a random variable that we exploit in this paper is that either it is almost surely constant (and equal to its expectation), or with positive probability it exceeds it expectation. More precisely, it is immediate from (1) and the definition of a probability that either $X(w)=\mathbf{E}_{P}(X)$ for all $w \in \sigma(P)$, or there is a value $w \in \sigma(P)$ with $X(w)>\mathbf{E}_{P}(X)$.

Suppose now that $\mathscr{A}=(Q, \Sigma)$ is an automaton with $|Q|=n$. We view elements of $\mathbb{R} Q$ as row vectors. Let $\pi: \mathbb{R} \Sigma \rightarrow M_{n}(\mathbb{R})$ be the corresponding matrix representation (cf. [27]); so if

$$
f=\sum_{w \in \Sigma^{*}} f(w) w
$$

and $q, r \in Q$, then

$$
\pi(f)_{q, r}=\sum_{\left\{w \in \Sigma^{*} \mid q w=r\right\}} f(w) .
$$

We shall usually omit $\pi$ from the notation and view $\mathbb{R} \Sigma$ as acting on row and column vectors. If $S \subseteq Q$, then $[S]$ denotes the characteristic row vector of $S$; e.g., $[Q]$ is the all ones row vector. We use $[S]^{T}$ to denote the transpose vector. A key fact is that $w[S]^{T}=\left[S w^{-1}\right]^{T}$ for $w \in \Sigma^{*}$, where as usual $S w^{-1}=\{q \in Q \mid q w \in S\}$.

Lemma 2 (Averaging Lemma) Let $\mathscr{A}=(Q, \Sigma)$ be a synchronizing automaton with $n$ states and let $P_{1}$ be a probability on $\Sigma^{*}$. Suppose that there exist $R \subseteq Q$ and a probability $P_{2}$ with support $\Sigma^{\leq n-1}$ such that:

1. $[R] P_{2} P_{1}=[R]$;

2. $R \subseteq q \Sigma^{*}$ for all $q \in R$;

3. there exists $w_{0} \in \Sigma^{*}$ with $Q w_{0} \subseteq R$.

Then $\mathscr{A}$ has a synchronizing word of length at most:

$-1+(n-2)(n-1+L)$ if $R=Q$;

$-(r-1)(n-1+L)+\ell$ if $R \subsetneq Q$

where $r=|R|, L$ is the maximum length of a word in $\sigma\left(P_{1}\right)$ and $\ell=\left|w_{0}\right|$.

Before, proving the lemma, let us use it to derive anew some results from the literature. The first is a result of Kari on synchronizing Eulerian automata [13]. An automaton is Eulerian if its underlying graph admits an Eulerian directed path, or equivalently, it is strongly connected and 
the in-degree of every vertex is the same as the out-degree (and hence is the alphabet size). Actually, we can generalize his result.

Let us say that a strongly connected automaton $\mathscr{A}=(Q, \Sigma)$ is pseudoEulerian if we can find a probability $P$ with support $\Sigma$ such that the matrix $\pi(P)$ is doubly stochastic (i.e., each row and column of $P$ adds up to 1). For instance, if $\mathscr{A}$ is Eulerian with adjacency matrix $A$ and $d=|\Sigma|$, then we can set

$$
P=\sum_{a \in \Sigma} d^{-1} a
$$

One checks that $\pi(P)=d^{-1} A$, and hence is doubly stochastic by the Eulerian hypothesis. Thus every Eulerian automaton is pseudo-Eulerian. It is easy to check whether a strongly connected automaton is pseudoEulerian: one just needs to look for a strictly positive solution to the system of $|Q|+1$ linear equations

$$
\begin{aligned}
1 & =\sum_{a \in \Sigma} p_{a} \\
1 & =\sum_{a \in \Sigma} p_{a} \cdot\left|q a^{-1}\right| \quad(q \in Q) .
\end{aligned}
$$

The automaton in Figure 1 is pseudo-Eulerian but not Eulerian. Indeed,

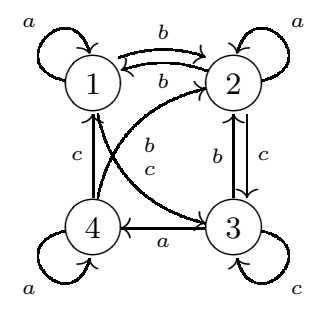

Fig. 1. A pseudo-Eulerian automaton

if we put $P=a / 2+b / 6+c / 3$, then

$$
\pi(B)=\left[\begin{array}{llll}
\frac{1}{2} & \frac{1}{6} & \frac{1}{3} & 0 \\
\frac{1}{6} & \frac{1}{2} & \frac{1}{3} & 0 \\
0 & \frac{1}{6} & \frac{1}{3} & \frac{1}{2} \\
\frac{1}{3} & \frac{1}{6} & 0 & \frac{1}{2}
\end{array}\right]
$$

is doubly stochastic. 
Theorem 3 An n-state synchronizing pseudo-Eulerian automaton has a synchronizing word of length at most $1+(n-2)(n-1)$.

Proof. Let $\mathscr{A}=(Q, \Sigma)$ and suppose that $P$ is a probability with support $\Sigma$ such that $\pi(P)$ is doubly stochastic. Let $P_{1}$ be the probability with support concentrated on the empty word and take $R=Q$ (and so $r=n$ ). As pseudo-Eulerian automata are strongly connected, $Q \subseteq q \Sigma^{*}$ for all $Q$. Put

$$
P_{2}=\frac{1}{n} \sum_{m=0}^{n-1} P^{m}
$$

it is a probability with support $\Sigma \leq n-1$. The condition that $\pi(P)$ is doubly stochastic is equivalent to $[Q] P=[Q]$. Thus

$$
[Q] P_{2} P_{1}=[Q] \cdot \frac{1}{n} \sum_{m=0}^{n-1} P^{m}=[Q] .
$$

The Averaging Lemma now yields the upper bound of $1+(n-2)(n-1)$ on the length of a synchronizing word.

The next result simultaneously generalizes results of Rystsov [7] on regular automata, Béal [24] on circular automata, Béal and Perrin [20] on one-cluster automata, and Carpi and d'Alessandro [21,22] on strongly and locally strongly transitive automata.

Theorem 4 Let $\mathscr{A}=(Q, \Sigma)$ be a synchronizing automaton. Suppose there is a set of words $W \subseteq \Sigma^{*}$ and $k \geq 1$ so that, for each state $q \in Q$ and each state $s \in R=Q W$, there are exactly $k$ elements of $W$ taking $q$ to $s$. Let $\ell$ be the length of the shortest word in $W$ and $L$ be the length of the longest. If $R=Q$, then there is a synchronizing word for $\mathscr{A}$ of length at most $1+(n-2)(n-1+L)$; if $R \subsetneq Q$, then there is a synchronizing word of length at most $(r-1)(n-1+L)+\ell$ where $r=|R|$.

Proof. A straightforward counting argument establishes that $|W|=k r$. It remains to define our probabilities in order to apply the Averaging Lemma. Take $P_{1}$ to be the uniform distribution on $W$ (so $P_{1}(w)=1 /|W|$ for $w \in W$ and is otherwise 0 ) and let $P_{2}$ be an arbitrary probability with support $\Sigma \leq n-1$. The only condition to check for the Averaging Lemma is that $[R] P_{2} P_{1}=[R]$. First observe that the columns of $\pi\left(P_{1}\right)$ corresponding to elements of $Q \backslash R$ are zero, while if $s \in R$, then the corresponding 
column of $\pi\left(P_{1}\right)$ is $k /|W|[Q]^{T}=1 / r[Q]^{T}$. Since $\pi\left(P_{2}\right)$ is a stochastic matrix (each of its rows sum to 1 ), this means that $\pi\left(P_{2} P_{1}\right)=\pi\left(P_{1}\right)$. Next observe that if $s \in R$, then $s \sum_{w \in W} w=k[R]$. Thus

$$
[R] \sum_{w \in W} w=\sum_{s \in R} s \sum_{w \in W} w=r k[R]=|W|[R] .
$$

Therefore, $[R] P_{1}=[R]$ and hence $[R] P_{2} P_{1}=[R]$, as required.

For example, Béal and Perrin [20] call $\mathscr{A}=(Q, \Sigma)$ a one-cluster automaton if there exists $a \in \Sigma$ so that $a$ has only one cycle $R$ on $Q$. Suppose that the cycle has size $r$. Then each state of $Q$ is taken to exactly one element of $R$ by the set of words $W=\left\{a^{n-r}, \ldots, a^{n-1}\right\}$. Theorem 4 then recovers the bound $1+2(n-2)(n-1)$ of [20]. Indeed, if $r=n$, this is direct. Otherwise, using $L=n-1$ and $\ell=n-r$, we obtain a bound of $(r-1)(2 n-2)+n-r=r(2 n-3)-n+2 \leq(n-1)(2 n-3)-n+2=$ $2 n^{2}-6 n+5=1+2(n-2)(n-1)$. Similarly, one recovers the results of Rystsov [7] and Carpi and d'Alessandro [21,22].

The proof of Theorem 4 can easily be adapted to obtain the same bound if $W$ is an arbitrary set of words such that there is a probability $P_{1}$ supported on $W$ so that each column of $\pi\left(P_{1}\right)$ corresponding to an element of $Q \backslash R$ is 0 , whereas each column corresponding to an element of $R$ is $1 / r[Q]^{T}$.

\section{Proof of the Averaging Lemma}

The proof of the Averaging Lemma rests on our observation about expectations of random variables and the ascending chain condition for finite dimensional vector spaces. Suppose that $\Sigma^{*}$ acts on the left of a vector space $V$ by linear maps. Let $X \subseteq \Sigma^{*}$ and let $W$ be a subspace. Then by $X W$, we mean the span of all vectors $x w$ with $x \in X$ and $w \in W$.

Lemma 5 Let $\pi: \Sigma^{*} \rightarrow M_{n}(K)$ be a matrix representation with $K$ a field. Suppose that one has subspaces $W, V \subseteq K^{n}$ of column vectors with $W \subseteq V$, but $\Sigma^{*} W \nsubseteq V$. Let $S$ be a spanning set for $W$. Then there exist $s \in S$ and $w \in \Sigma^{*}$ with $|w| \leq \operatorname{dim} V-\operatorname{dim} W+1$ and $w s \notin V$.

Proof. Put $W_{m}=\Sigma^{\leq m} W$. Then there is an ascending chain of subspaces

$$
W=W_{0} \subseteq W_{1} \subseteq W_{2} \subseteq \cdots
$$


and moreover as soon as this chain stabilizes it equals $\Sigma^{*} W$. By our assumption, there is a greatest $m \geq 0$ with $W_{m} \subseteq V$. In particular, the chain does not stabilize until after $m$ steps and so

$$
W_{0} \subsetneq W_{1} \subsetneq \cdots \subsetneq W_{m} \subseteq V
$$

and hence $\operatorname{dim} W_{0}+m \leq \operatorname{dim} V$, that is, $m+1 \leq \operatorname{dim} V-\operatorname{dim} W+1$. Therefore, there is a word $w \in \Sigma^{*}$ with $|w| \leq \operatorname{dim} V-\operatorname{dim} W+1$ and $w W \nsubseteq V$. But $W$ is spanned by $S$, so we can find $s \in S$ with $w s \notin V$.

Proof (of the Averaging Lemma). For convenience, put $X=\sigma\left(P_{1}\right)$. We show that for each $\emptyset \neq S \subsetneq R$, there exists $w \in \Sigma^{*}$ of length at most $n-1+L$ with $\left|S w^{-1} \cap R\right|>|S|$. If $R=Q$, the result is then immediate: one can find a state $q \in Q$ and a letter $a \in \Sigma$ so that $\left|q a^{-1}\right|>1$; now we expand by inverse images $n-2$ times with words of length at most $n-1+L$ to obtain the result. If $R \subsetneq Q$, we can find $w$ of length at most $(r-1)(n-1+L)$ with $|R w|=1$ using the same idea. Then as $Q w_{0} \subseteq R$, it follows $\left|Q w_{0} w\right| \leq|R w|=1$. This yields the bound of $(r-1)(n-1+L)+\ell$ on the length a synchronizing word.

Consider the probability $P=P_{2} P_{1}$ on $\Sigma^{*}$ and define a random variable $Z_{S}: \Sigma^{*} \rightarrow \mathbb{R}$ by

$$
Z_{S}(w)=\left|S w^{-1} \cap R\right|=[R]\left[S w^{-1}\right]^{T}=[R][w][S]^{T} .
$$

Let us compute the expected value of this random variable:

$$
\begin{aligned}
\mathbf{E}_{P}\left(Z_{S}\right)= & \sum_{w \in \Sigma^{*}} P(w)\left|S w^{-1} \cap R\right|=\sum_{w \in \Sigma^{*}} P(w)[R] w[S]^{T} \\
& =[R] P[S]^{T}=[R] P_{2} P_{1}[S]^{T}=[R][S]^{T} \\
& =|S|
\end{aligned}
$$

where we have used $[R] P_{2} P_{1}=[R]$. The support of $P$ is $\sigma\left(P_{2}\right) \sigma\left(P_{1}\right)=$ $\Sigma^{\leq n-1} X$. So if we can find $v \in \Sigma^{\leq n-1} X$ with $Z_{S}(v)=\left|S v^{-1} \cap R\right| \neq|S|$, then we can find $w \in \Sigma^{\leq n-1} X$ with $\left|S w^{-1} \cap R\right|=Z_{S}(w)>0$ by our discussion earlier on random variables that are not almost surely constant. As $|w| \leq n-1+L$, this will finish the proof.

If $\left|S x^{-1} \cap R\right| \neq|S|$ for some $x \in X$, then we are done. Otherwise, we may assume $\left|S x^{-1} \cap R\right|=|S|$ for all $x \in X$. Let $\gamma$ be the column vector $[S]^{T}-|S| / r[Q]^{T}$. Notice that if $w \in \Sigma^{*}$, then $w \gamma=\left[S w^{-1}\right]^{T}-|S| / r[Q]^{T}$ and so $[R] w \gamma=\left|S w^{-1} \cap R\right|-|S|$. In particular, if $x \in X$ our assumption implies $[R] x \gamma=0$. Moreover, $x \gamma \neq 0$ as $|S|<r$. Thus if $W$ is the subspace spanned by the column vectors $x \gamma$ with $x \in X$, then $0 \neq W \subseteq[R]^{\perp}$. 
We claim that $\Sigma^{*} W \nsubseteq[R]^{\perp}$. Indeed, let $w$ be a synchronizing word. Then $w w_{0}$ synchronizes $\mathscr{A}$ to an element of $q \in R$. But $q \Sigma^{*} \supseteq R$, so we can synchronize to any state of $R$. In particular, we can synchronize $\mathscr{A}$ via some word $y$ into $S x^{-1} \cap R$ for some $x \in X$. Then $S x^{-1} y^{-1}=Q$ and so $[R] y x \gamma=\left|S x^{-1} y^{-1} \cap R\right|-|S|>0$. This shows that $y x \gamma \notin R^{\perp}$ and hence $\Sigma^{*} W \nsubseteq[R]^{\perp}$. As $\operatorname{dim} W \geq 1$ and $\operatorname{dim}[R]^{\perp}=n-1$, Lemma[5]now provides $u \in \Sigma^{\leq n-1}$ and $z \in X$ with $u z \gamma \notin[R]^{\perp}$. Putting $v=u z \in \Sigma^{\leq n-1} X$, we have $0 \neq[R] v \gamma=\left|S v^{-1} \cap R\right|-|S|$. This completes the proof.

\section{References}

1. Černý, J.: A remark on homogeneous experiments with finite automata. Mat.-Fyz. Časopis Sloven. Akad. Vied 14 (1964) 208-216

2. Pin, J.E.: Sur un cas particulier de la conjecture de Cerny. In: Automata, languages and programming (Fifth Internat. Colloq., Udine, 1978). Volume 62 of Lecture Notes in Comput. Sci. Springer, Berlin (1978) 345-352

3. Pin, J.E.: Le problème de la synchronisation et la conjecture de Černý. In: Noncommutative structures in algebra and geometric combinatorics (Naples, 1978). Volume 109 of Quad. "Ricerca Sci.". CNR, Rome (1981) 37-48

4. Arnold, F., Steinberg, B.: Synchronizing groups and automata. Theoret. Comput. Sci. 359 (2006) 101-110

5. Dubuc, L.: Sur les automates circulaires et la conjecture de Černý. RAIRO Inform. Théor. Appl. 32 (1998) 21-34

6. Ananichev, D.S., Volkov, M.V., Zaks, Y.I.: Synchronizing automata with a letter of deficiency 2. Theoret. Comput. Sci. 376 (2007) 30-41

7. Rystsov, I.K.: Quasioptimal bound for the length of reset words for regular automata. Acta Cybernet. 12 (1995) 145-152

8. Rystsov, I.K.: On the length of reset words for automata with simple idempotents. Kibernet. Sistem. Anal. (2000) 32-39, 187

9. Almeida, J., Margolis, S., Steinberg, B., Volkov, M.: Representation theory of finite semigroups, semigroup radicals and formal language theory. Trans. Amer. Math. Soc. 361 (2009) 1429-1461

10. Trahtman, A.N.: The Černý conjecture for aperiodic automata. Discrete Math. Theor. Comput. Sci. 9 (2007) 3-10 (electronic)

11. Trahtman, A.N.: An efficient algorithm finds noticeable trends and examples concerning the Cerny conjecture. In: Mathematical foundations of computer science 2006. Volume 4162 of Lecture Notes in Comput. Sci. Springer, Berlin (2006) 789800

12. Ananichev, D.S., Volkov, M.V.: Some results on Černý type problems for transformation semigroups. In: Semigroups and languages. World Sci. Publ., River Edge, NJ (2004) 23-42

13. Kari, J.: Synchronizing finite automata on Eulerian digraphs. Theoret. Comput. Sci. 295 (2003) 223-232 Mathematical foundations of computer science (Mariánské Lázně, 2001).

14. Ananichev, D.S., Volkov, M.V.: Synchronizing generalized monotonic automata. Theoret. Comput. Sci. 330 (2005) 3-13

15. Rystsov, I.: Reset words for commutative and solvable automata. Theoret. Comput. Sci. 172 (1997) 273-279 
16. Rystsov, I.C.: On the rank of a finite automaton. Kibernet. Sistem. Anal. (1992) 3-10, 187

17. Steinberg, B.: Černý's conjecture and group representation theory. J. Algebr. Comb. (2008)

18. Kari, J.: A counter example to a conjecture concerning synchronizing words in finite automata. Bull. Eur. Assoc. Theor. Comput. Sci. EATCS (2001) 146

19. Volkov, M.V.: Synchronizing automata and the Cerný conjecture. In MartínVide, C., Otto, F., Fernau, H., eds.: Language and Automata Theory and Applications Second International Conference, LATA 2008, Tarragona, Spain, March 13-19, 2008. Volume 5196 of Lecture Notes in Computer Science., Berlin / Heidelberg, Springer (2008) 11-27

20. Béal, M.P., Perrin, D.: A quadratic upper bound on the size of a synchronizing word in one-cluster automata. In: Developments in language theory. Volume 5583 of Lecture Notes in Comput. Sci. Springer, Berlin (2009) 81-90

21. Carpi, A., d'Alessandro, F.: The synchronization problem for strongly transitive automata. In: Developments in language theory. Volume 5257 of Lecture Notes in Comput. Sci. Springer, Berlin (2008) 240-251

22. Carpi, A., d'Alessandro, F.: The synchronization problem for locally strongly transitive automata. In: Mathematical Foundations of Computer Science. Volume 5734 of Lecture Notes in Comput. Sci. Springer, Berlin (2009) 211-222

23. Almeida, J., Steinberg, B.: Matrix mortality and the Cerný-Pin conjecture. In: Developments in language theory. Volume 5583 of Lecture Notes in Comput. Sci. Springer, Berlin (2009) 67-80

24. Béal, M.P.: A note on Cerny's conjecture and rational series. Unpublished (2003)

25. Salomaa, A.: Composition sequences for functions over a finite domain. Theoret. Comput. Sci. 292 (2003) 263-281 Selected papers in honor of Jean Berstel.

26. Pin, J.E.: On two combinatorial problems arising from automata theory. In: Combinatorial mathematics (Marseille-Luminy, 1981). Volume 75 of North-Holland Math. Stud. North-Holland, Amsterdam (1983) 535-548

27. Berstel, J., Reutenauer, C.: Rational series and their languages. Volume 12 of EATCS Monographs on Theoretical Computer Science. Springer-Verlag, Berlin (1988) 